\title{
Prospecção fitoquímica, teor de fenóis totais e atividade antiradicalar de extrato e frações de folhas de Annona sylvatica (Annonaceae)
}

\author{
Phytochemical prospection, total phenol content and anti-radical activity of leaf extract and \\ fractions of Annona sylvatica (Annonaceae) \\ Prospección fitoquímica, contenido total de fenoles y actividad antirradicales del extracto de hoja y \\ fracciones de Annona sylvatica (Annonaceae)
}

Recebido: 04/11/2021 | Revisado: 15/11/2021 | Aceito: 17/11/2021 | Publicado: 27/11/2021

\author{
Tamara Mendes Leite Silva Trindade \\ ORCID: https://orcid.org/0000-0001-7082-2227 \\ Universidade Federal do Tocantins, Brasil \\ E-mail: tamara_trindade@live.com \\ Almir Ribeiro de Carvalho Junior \\ ORCID: https://orcid.org/0000-0001-9821-4004 \\ Instituto Federal da Bahia, Brasil \\ E-mail: almir@uenf.br \\ Rafaela Oliveira Ferreira \\ ORCID: https://orcid.org/0000-0003-1637-8393 \\ Universidade Federal do Recôncavo da Bahia, Brasil \\ E-mail: rafaelaoliveira@ufrb.edu.br
}

\begin{abstract}
Resumo
O consumo de plantas medicinais tem apresentado um crescimento considerável em diversos países. Annona sylvatica, conhecida vulgarmente como araticum-do-mato, é uma frutífera empregada na medicina popular para baixar a pressão arterial. O objetivo deste trabalho foi investigar o perfil fitoquímico e avaliar a atividade antioxidante de extrato e frações de folhas de A. sylvatica. $\mathrm{O}$ extrato em etanol foi obtido por maceração e submetido à partição líquido-líquido para obtenção das frações em hexano, clorofórmio e acetato de etila. As principais classes de metabólitos secundários foram detectadas por prospecção fitoquímica, o teor de fenóis totais (FT) e a atividade antioxidante foram quantificados pelos testes de Folin-Dennis e ensaio da atividade sequestradora do radical DPPH, respectivamente. Através do coeficiente de Pearson, os teores de fenóis totais foram correlacionados com os valores de $\mathrm{CE}_{50}$ observados no ensaio do DPPH. A prospecção fitoquímica detectou a presença de flavonoides, saponinas, alcaloides e triterpenos/esteroides. A fração AcOET apresentou o maior teor de fenóis totais e maior atividade no ensaio do DPPH, com os valores de 84,0 44,4 mg EAG. ${ }^{-1}$ e CE50 9,28 $\mu \mathrm{g}^{\mathrm{mL}} \mathrm{mL}^{-1}$, respectivamente. Observou-se uma forte correlação negativa entre o teor de FT e os valores de $\mathrm{CE}_{50}$, sugerindo que parte da atividade antioxidante observada está relacionada ao teor de FT. Os resultados sugerem que a araticum-do-mato é uma importante fonte de antioxidantes naturais.
\end{abstract}

Palavras-chave: Araticum-do-mato; Fitoquímica; Flavonoides; DPPH; Antioxidantes.

\begin{abstract}
The consumption of medicinal plants has shown considerable growth in several countries. Annona sylvatica, commonly known as araticum-do-mato, is a fruit used in folk medicine to lower blood pressure. The objective of this work was to investigate the phytochemical profile and evaluate the antioxidant activity of $A$. sylvatica leaf extracts and fractions. The ethanol extract was obtained by maceration and submitted to liquid-liquid partition to obtain fractions in hexane, chloroform and ethyl acetate. The main classes of secondary metabolites were detected by phytochemical screening, the content of total phenols (TF) and the antioxidant activity were quantified by the FolinDennis tests and the DPPH radical scavenging activity assay, respectively. Using the Pearson coefficient, the total phenol contents were correlated with the $\mathrm{EC}_{50}$ values observed in the DPPH assay. Phytochemical screening detected the presence of flavonoids, saponins, alkaloids and triterpenes/steroids. The AcOET fraction showed the highest content of total phenols and the highest activity in the DPPH assay, with values of 84.0 $\pm 4.4 \mathrm{mg}$ GAE. $\mathrm{g}^{-1}$ and EC 50 $9.28 \mu \mathrm{g} . \mathrm{mL}^{-1}$, respectively. There was a strong negative correlation between $\mathrm{TF}$ content and $\mathrm{EC}_{50}$ values, suggesting that part of the antioxidant activity observed is related to TF content. The results suggest that araticum-do-mato is an important source of natural antioxidants.
\end{abstract}

Keywords: Araticum-do-mato; Phytochemistry; Flavonoids; DPPH; Antioxidants. 


\begin{abstract}
Resumen
El consumo de plantas medicinales ha mostrado un crecimiento considerable en varios países. Annona sylvatica, comúnmente conocida como araticum-do-mato, es una fruta que se usa en la medicina popular para reducir la presión arterial. El objetivo de este trabajo fue investigar el perfil fitoquímico y evaluar la actividad antioxidante de extractos y fracciones de hojas de A. sylvatica. El extracto etanólico se obtuvo por maceración y se sometió a reparto líquidolíquido para obtener fracciones en hexano, cloroformo y acetato de etilo. Las principales clases de metabolitos secundarios se detectaron mediante cribado fitoquímico, el contenido de fenoles totales (TF) y la actividad antioxidante se cuantificaron mediante las pruebas de Folin-Dennis y el ensayo de actividad captadora de radicales DPPH, respectivamente. Usando el coeficiente de Pearson, el contenido total de fenol se correlacionó con los valores de $\mathrm{CE}_{50}$ observados en el ensayo DPPH. El cribado fitoquímico detectó la presencia de flavonoides, saponinas, alcaloides y triterpenos/esteroides. La fracción AcOET mostró el mayor contenido de fenoles totales y la mayor actividad en el ensayo DPPH, con valores de 84,0 $\pm 4,4 \mathrm{mg}$ EAG. $\mathrm{g}^{-1}$ y EC 50 9,28 $\mu \mathrm{g}$. $\mathrm{mL}^{-1}$, respectivamente. Se observó una fuerte correlación negativa entre el contenido de TF y los valores de $\mathrm{CE}_{50}$, lo que sugiere que parte de la actividad antioxidante observada está relacionada con el contenido de TF. Los resultados sugieren que araticum-domato es una fuente importante de antioxidantes naturales.
\end{abstract}

Palabras clave: Araticum-do-mato; Fitoquímica; Flavonoides; DPPH; Antioxidantes.

\title{
1. Introdução
}

O uso dos produtos naturais iniciou-se há milhares de anos por populações de vários países com o intuito de tratar diversas patologias. Sendo empregados pela população como forma alternativa ou complementar aos medicamentos sintéticos (Chen et al., 2016). Plantas e fungos forneceram e/ou inspiraram produtos farmacêuticos essenciais para o tratamento de diversas doenças, como o câncer, as doenças cardíacas, a demência, a malária, dentre outras (Howes et al., 2020). Na área de tratamento do câncer, por exemplo, entre as 185 moléculas de baixo peso molecular introduzidos no mercado mundial no período de 1981-2019, 64,9 \% são substâncias derivadas ou inspiradas em produtos naturais (Newman e Cragg, 2020).

A família Annonaceae é representada no Brasil por cerca de 386 espécies, sendo 158 endêmicas, predominantes na região Amazônica (Lobão et al., 2012). O gênero Annona compreende cerca de 119 espécies, distribuídas na América e África tropicais (Pinto et al., 2005). Espécies deste gênero são conhecidas pela produção de frutos comestíveis e por suas propriedades medicinais, sendo utilizadas na medicina popular para o tratamento de diversas doenças, como antiparasitário, inflamações, diabetes e câncer (Mishra et al., 2013; Quílez et al., 2018; Leite et al., 2020). O seu uso medicinal motivou a realização de vários estudos farmacológicos com extratos, frações e substâncias isoladas destas espécies, sendo verificadas suas propriedades antitumorais, anti-inflamatórias, antioxidantes, antimicrobianas, antiparasitárias, citotóxicas, dentre outras. Quimicamente, espécies de Annona são caracterizadas pela biossíntese de acetogeninas, alcaloides, flavonoides, triterpenos, esteroides e óleos essenciais (Quílez et al., 2018; Leite et al., 2020).

Annona sylvatica, sinonímia Rollinia sylvatica, conhecida vulgarmente como coresma e araticum-do-mato, produz frutos comestíveis e é empregada na medicina popular para baixar a pressão arterial (Vendruscolo e Mentz, 2006). Estudos farmacológicos com extratos de diferentes partes desta planta indicaram atividades anti-inflamatória, anticancerígena, antimicrobiana, antioxidante e inseticida (Formagio et al., 2013; Araújo et al., 2014; Benites et al., 2015; Gonçalves et al., 2015). O óleo essencial de folhas mostrou-se rico em sesquiterpenos (Formagio et al., 2013), na polpa dos frutos e sementes detectaram-se fenóis, flavonoides e vitamina C (Benites et al., 2015) e o estudo fitoquímico da fração em acetato de etila de folhas conduziu a identificação da quercetina, luteolina e almunequina (Araújo et al., 2014). Apesar do seu uso alimentício e medicinal, poucos relatos de estudos químico-farmacológicos foram descritos para a espécie. Desta forma, o presente trabalho propõe realizar a prospecção fitoquímica, quantificar o teor de fenóis totais e avaliar a atividade antioxidante de extratos e frações de folhas de A. sylvatica coletadas no estado do Tocantins. 


\section{Metodologia}

\subsection{Materiais e procedimentos gerais}

Ácido ascórbico, ácido gálico, 2,2-difenil-1-picril-hidrazila (DPPH), reagente de Folin-Denis e carbonato de sódio foram adquiridos pela Merck e Vetec. Solventes P.A. foram adquiridos pela Quimex e Nox Chemicals. Extratos e frações foram concentrados em evaporador rotativo Fisatom 802. Análises fotométricas foram registradas em espectrofotômetro UVVIS T60 PG instruments.

\subsection{Coleta do material vegetal e preparação de extratos}

Folhas de A. sylvatica foram coletados no campus da Universidade Federal do Tocantins, Gurupi -TO. Uma exsicata foi depositada no Herbário do Recôncavo da Bahia HURB - UFRB (número 26018). Folhas secas (300,0 g) foram triturados em liquidificador e submetidos a maceração exaustiva em EtOH em condições ambiente. O material extraído foi filtrado e concentrado em rotaevaporador, fornecendo o extrato bruto em EtOH (código ASE). Parte do extrato ASE (10,0 g) foi dissolvido em uma solução de $\mathrm{MeOH}: \mathrm{H}_{2} \mathrm{O}$ (3:7) e submetido a partição líquido-líquido em funil de separação empregando-se solventes em polaridades crescentes, sendo obtidas as frações em hexano (4,45 g, código ASH), clorofórmio (2,90 g, código ASC) e acetato de etila (1,65 g, código ASAE).

\subsection{Prospecção fitoquímica}

O extrato e as frações orgânicas de folhas de A. sylvatica foram submetidos à triagem fitoquímica preliminar visando verificar qualitativamente a presença dos principais grupos químicos naturais. As análises foram realizadas de acordo com Matos (1997), observou-se a formação de espuma, reações colorimétricas e/ou de precipitação para determinar a ausência e/ou presença das substâncias. As classes de metabólitos secundários investigadas foram: taninos (precipitação de proteínas, teste da gelatina), saponinas (formação de espuma), triterpenos/esteroides (reação de Liebermann-Burchard), alcaloides (reação de Dragendorff), antocianinas, antocianidinas, flavonoides, chalconas e xantonas (coloração das amostras em pH 3, 8 e 11), leucoantocianidinas, catequinas e flavanonas (aquecimento e coloração das amostras em pH 3 e 11). As análises fitoquímicas foram realizadas em três repetições e os resultados, mudança de coloração, formação de espuma e/ou precipitados, foram comparados com o controle (somente extrato e/ou fração).

\subsection{Teor de fenóis totais}

O teor de fenóis totais (FT) foi determinado pelo método de Folin-Dennis (Singleton et al., 1999), com modificações. A 2,5 mL do reagente de Folin-Dennis adicionou-se uma alíquota de $0,5 \mathrm{~mL}$ de solução de extrato e/ou frações $\left(1,0 \mathrm{mg}\right.$. $\left.\mathrm{mL}^{-1}\right)$ e após 5 minutos adicionou-se $2,0 \mathrm{~mL}$ de solução aquosa de $\mathrm{Na}_{2} \mathrm{CO}_{3} 14 \%$. Após duas horas, as absorbâncias foram lidas a 760 $\mathrm{nm}$ em um espectrofotômetro UV-VIS. Os ensaios foram realizados em três repetições. O teor de FT foi quantificado a partir de uma curva de calibração construída com o padrão ácido gálico $\left(0-0,022 \mathrm{mg} \cdot \mathrm{mL}^{-1}\right)$. Os resultados foram expressos como miligramas de equivalente ao ácido gálico por grama de extrato (mg EAG. $\mathrm{g}^{-1}$ ).

\subsection{Atividade sequestradora do radical livre DPPH}

O ensaio do DPPH foi realizado de acordo com a metodologia proposta por Sousa et al. (2007), com modificações. Adicionou-se uma alíquota de 2,7 mL de solução de DPPH $\left(40 \mu \mathrm{g} . \mathrm{mL}^{-1}\right)$ a $0,3 \mathrm{~mL}$ de diferentes concentrações de extratos e/ou frações (1 a $200 \mu \mathrm{g}$. $\left.\mathrm{mL}^{-1}\right)$ e do padrão ácido ascórbico (1 a $\left.10 \mu \mathrm{g} \cdot \mathrm{mL}^{-1}\right)$. Após 30 minutos, as absorbâncias foram lidas a 
$517 \mathrm{~nm}$ em um espectrofotômetro UV-VIS. Utilizou-se uma mistura de DPPH (2,7 mL) e metanol (0,3 mL) como controle negativo. A atividade sequestradora (\% AS) foi determinada pela equação 1 :

$$
\% \mathrm{AS}=100 \times\left(\mathrm{A}_{\text {controle }}-\mathrm{A}_{\text {amostra }}\right) / \mathrm{A}_{\text {controle }}
$$

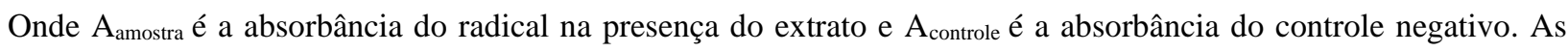
análises foram efetuadas em três repetições A eficiência antirradicalar foi determinada por regressão linear no intervalo de confiança de $95 \%(\mathrm{p}<0,05)$. Os resultados foram descritos em $\mathrm{CE}_{50}$, que corresponde a concentração da amostra necessária para capturar $50 \%$ dos radicais livres de DPPH.

\subsection{Análises estatísticas}

As análises foram realizadas em três repetições, com os valores expressos em média \pm desvio padrão. As médias foram comparadas pelo teste de Tukey. A correlação entre o teor de FT e os valores de $\mathrm{CE}_{50}$ foi avaliada de acordo com a abordagem clássica para distribuição de Pearson com a utilização dos seguintes critérios: $|r|<0,3$ (correlação fraca), 0,3 $\leq|r|<0,6$ (correlação moderada) e $|\mathrm{r}| \geq 0,6$ (correlação forte). As análises estatísticas foram feitas no programa GraphPad 5.0 DEMO.

\section{Resultados e Discussão}

\subsection{Prospecção fitoquímica}

O uso de plantas medicinais está presente na terapêutica popular mundial. A utilização de métodos qualitativos de baixo custo como a prospecção fitoquímica é importante como screening inicial na triagem de espécies de importância farmacológica e/ou toxicológica, especialmente aquelas oriundas de biomas de expresso interesse para preservação da biodiversidade como o Cerrado (Matos, 1997; Bessa et al., 2013). Os dados da triagem fitoquímica de extrato e frações orgânicas de folhas de A. sylvatica estão descritos na Tabela 1 e Figura 1.

Tabela 1. Prospecção fitoquímica de extrato e frações de folhas de A. sylvatica.

\begin{tabular}{|c|c|c|c|c|}
\hline Classes de metabólitos secundários & ASE & ASH & $\overline{\mathrm{ASC}}$ & ASAE \\
\hline Esteroides e triterpenos & + & + & + & - \\
\hline Saponinas & + & - & - & - \\
\hline Alcaloides & + & - & - & + \\
\hline Taninos & - & - & - & - \\
\hline Antocianinas e antocianidinas & - & - & - & - \\
\hline Flavonas, flavonóis e xantonas & + & - & + & - \\
\hline Chalconas e auronas & - & - & - & - \\
\hline Flavanonóis & - & - & + & + \\
\hline Leucoantocianidinas & - & - & - & - \\
\hline Catequinas & - & - & - & - \\
\hline Flavanonas & - & - & - & + \\
\hline
\end{tabular}

(+) Detectado; (-) Não detectado. Fonte: Autores (2021). 
Figura 1. Ensaios de prospecção fitoquímica de extrato e frações de folhas de A. sylvatica.

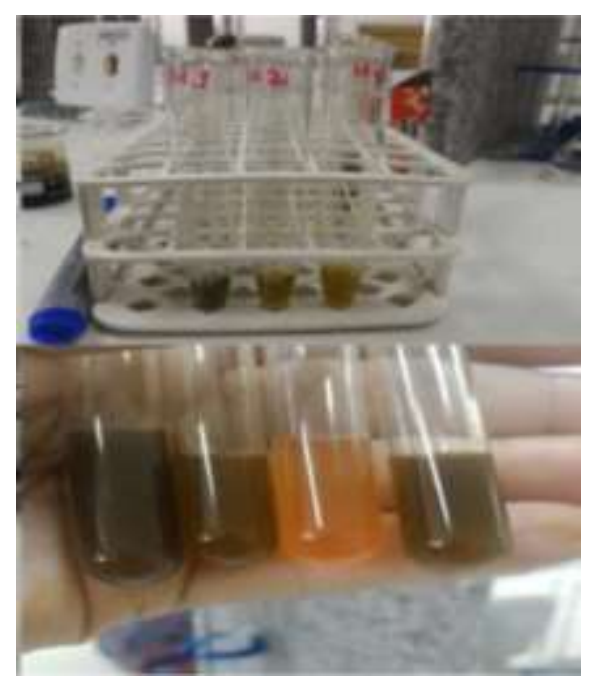

Fonte: Autores (2017).

A triagem fitoquímica mostrou a eficiência do fracionamento do extrato ASE de folhas de A. sylvatica por partição com solventes de polaridades crescentes (Tabela 01). De acordo com Simões et al. (2016), a realização de caracterização dos grupos de substâncias vegetais diretamente no extrato bruto pode eventualmente mascarar o resultado, os ensaios com as frações orgânicas possibilitam reações mais nítidas. O etanol possibilita a extração da maioria das substâncias de interesse para a análise fitoquímica, inclusive os heterosídeos. O acetato de etila é eficiente na extração de flavonoides e cumarinas, o clorofórmio em antraquinonas, alcaloides e glicosídeos cardiotônicos, já o hexano na extração de lipídeos, ceras, pigmentos e furanocumarinas (Simões et al., 2016).

Os alcaloides foram detectados no extrato em EtOH e na fração em acetato de etila de folhas de A. sylvatica. Os alcaloides formam sais duplos com metais, como o ouro, a platina e o mercúrio. Usualmente, esses sais são obtidos na forma de complexos insolúveis usados na caracterização desta classe de metabólitos (Simões et al., 2016). Espécies de Annona são conhecidas pela biossíntese de alcaloides (Quílez et al., 2018). Várias atividades farmacológicas são atribuídas aos alcaloides, como antimicrobiana e anti-inflamatória (Carvalho Junior et al., 2019).

Os flavonoides foram detectados no extrato em EtOH e nas frações em clorofórmio e acetato de etila de folhas de A. sylvatica. Os flavonoides são sensíveis a mudanças de $\mathrm{pH}$ do meio, sendo suscetíveis a hidrólise ácida e básica. A identificação dos diferentes núcleos flavônicos foi realizada com base nas diferenças de solubilidade e nas reações de coloração (Março e Poppi, 2008; Paz et al., 2014). A análise de frações de folhas de A. sylvatica por cromatografia líquida acoplada a espectrometria de massas (CL-EM) conduziu a identificação dos flavonoides quercetina, luteolina e almunequina (Araújo et al., 2014), corroborando com os resultados observados neste estudo. Os flavonoides são uma importante classe de substâncias bioativas, vários estudos in vivo e in vitro comprovam seu potencial antioxidante, antitumoral, antinociceptivo, anti-inflamatório, dentre outros (Ferreira et al., 2016).

As saponinas foram detectadas no extrato em EtOH de folhas de A. sylvatica a partir da formação de espuma persistente, o efeito espumante é devido as suas propriedades emulsificantes e da diminuição da tensão superficial do líquido (Azevedo et al., 2014). As saponinas foram identificadas na prospecção fitoquímica de extratos de folhas de outras espécies de Annona, como A. reticulata (Babu e Lawrence, 2014), A. squamosa (Neethu Simon et al., 2016) e A. senegalensis (Ijaiya et al., 2014). 
Triterpenos e esteroides foram identificados no extrato em EtOH e nas frações em hexano e clorofórmio. Neste ensaio, ocorrem reações de desidratação e oxidação do sistema de anéis formando o esteroide e/ou triterpeno aromático responsável pela coloração esverdeada observada no ensaio positivo (Paz et al., 2014). Os triterpenos e esteroides são conhecidos por suas propriedades anti-inflamatórias, antioxidantes e antitumorais (Vechia et al., 2009). Este é o primeiro estudo a relatar a prospecção fitoquímica de extratos de folhas de A. sylvatica, a presença destes grupos de metabólitos secundários pode contribuir para as propriedades medicinais atribuídas a esta espécie e estão de acordo com o perfil químico descritos para espécies deste gênero (Quílez et al., 2018).

\subsection{Teor de fenóis totais e atividade antioxidante}

O excesso de radicais livres está associado a diversas doenças, como o câncer, doenças cardiovasculares, doenças neurológicas, dentre outras. Dietas ricas em frutas e vegetais, com compostos antioxidantes, geralmente estão relacionadas a uma menor incidência de doenças relacionadas ao estresse oxidativo. Flavonoides e compostos fenólicos em geral são poderosos antioxidantes, capazes de captar radicais livres prevenindo ou atenuando suas ações deletérias no organismo (Martins et al., 2018; Vetrania et al., 2020). O ensaio da atividade sequestradora do radical DPPH consiste em um método simples, rápido e reprodutível para analisar a atividade antioxidante de extratos vegetais e substâncias naturais (Alves et al., 2010). O teor de fenóis totais do extrato e frações de folhas de A. sylvatica quantificados pela metodologia de Folin-Dennis e atividade sequestradora do radical DPPH estão descritos na Tabela 2.

Tabela 2. Teor de fenóis totais e atividade antioxidante de extrato e frações de folhas de A. sylvatica.

\begin{tabular}{lcc}
\hline \multicolumn{1}{c}{ Extrato, frações, padrão } & $\begin{array}{c}\text { Teor de fenóis totais } \\
\left(\mathbf{m g ~ E A G . ~} \mathbf{g}^{-\mathbf{1}}\right)\end{array}$ & $\begin{array}{c}\text { DPPH } \\
\left(\mathbf{C E} \mathbf{5 0} \boldsymbol{\mu g} \cdot \mathbf{~ m L}^{\mathbf{1}}\right)\end{array}$ \\
\hline ASE & $22,74 \pm 1,5$ & $74,48 \pm 0,6$ \\
ASH & $13,23 \pm 1,6$ & $68,29 \pm 4,5$ \\
ASC & $8,19 \pm 0,5$ & $64,88 \pm 1,9$ \\
ASAE & $84,0 \pm 4,4$ & $9,28 \pm 0,1$ \\
Ácido ascórbico & & $2,01 \pm 0,1$ \\
\hline
\end{tabular}

Fonte: Autores (2017).

A fração em AcOEt de folhas de A. sylvatica mostrou o maior teor de FT $\left(84,0 \pm 4,4\right.$ mg EAG. $\left.\mathrm{g}^{-1}\right)$ em comparação as demais amostras analisadas $(\mathrm{p}<0,05)$. O teor de FT para os extratos hidrometanólicos da polpa dos frutos e das sementes de $A$.

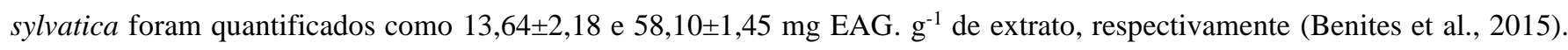
Extratos em MeOH e em EtOH das folhas de A. squamosa apresentaram teores de FT de 117,2 e 112, 9 mg EAG. $\mathrm{g}^{-1}$, respectivamente (Ibrahim et al., 2020). O extrato etanólico e as frações hexânica e $\mathrm{CHCl}_{3}$ das folhas de A. vepretorum

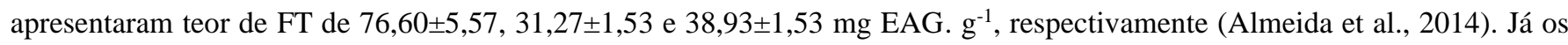
extratos em hexano e metanol das folhas do híbrido atemoia (A. cherimola Mill x A. squamosa L.) apresentaram os teores de $74,47 \pm 7,50$ e 75 $\pm 2,64$ mg EAG. $\mathrm{g}^{-1}$, respectivamente (Rabêlo et al., 2014). Estes resultados sugerem que a quantificação do teor de FT pode variar dentro de uma mesma espécie dependendo do órgão analisado e também está relacionada a polaridade do solvente extrator. Considerando os resultados descritos observamos que os extratos de folhas de A. sylvatica apresentaram um moderado teor de FT em comparação a outras espécies do gênero Annona.

$\mathrm{O}$ extrato e frações de folhas de A. sylvatica apresentaram atividade sequestradora de radical livre com $\mathrm{CE}_{50}$ variando de $9,28 \pm 0,1$ a 74,48 $\pm 0,6 \mu \mathrm{g}$. $\mathrm{mL}^{-1}$ (Figura 2). A fração em AcOEt apresentou a maior atividade antioxidante dentre as amostras 
analisadas $\left(\mathrm{CE}_{50}=9,28 \pm 0,1 \mu \mathrm{g} . \mathrm{mL}^{-1}\right)$, entretanto foi menos ativa que o padrão ácido ascórbico (Teste de Tukey, p<0,05). O Coeficiente de correlação de Pearson $r=-0,954$ indicou uma forte correlação negativa entre o teor de fenóis totais e os valores de $\mathrm{CE}_{50}$, indicando que quanto maior o teor de fenóis totais maior a atividade sequestradora do radical DPPH das amostras testadas. Esta análise sugere que a atividade antioxidante está relacionada ao teor de fenóis presentes no extrato e frações de folhas de A. sylvatica. Extratos de folhas de outras espécies do gênero Annona (ex. A. squamosa, A. vepretorum e A. cherimola $x$ A. squamosa) exibiram atividade antioxidante no ensaio com DPPH com valores de $\mathrm{CE}_{50}$ variando entre $13,61 \mathrm{e}$ 98,87 mg. $\mathrm{mL}^{-1}$ (Almeida et al., 2014; Rabêlo et al., 2014; Ibrahim et al., 2020).

Figura 2. Percentagem de atividade sequestradora do DPPH em relação a concentração em $\mu \mathrm{g}$. $\mathrm{mL}^{-1}$ das amostras de extratos e frações de folhas de A. sylvatica.
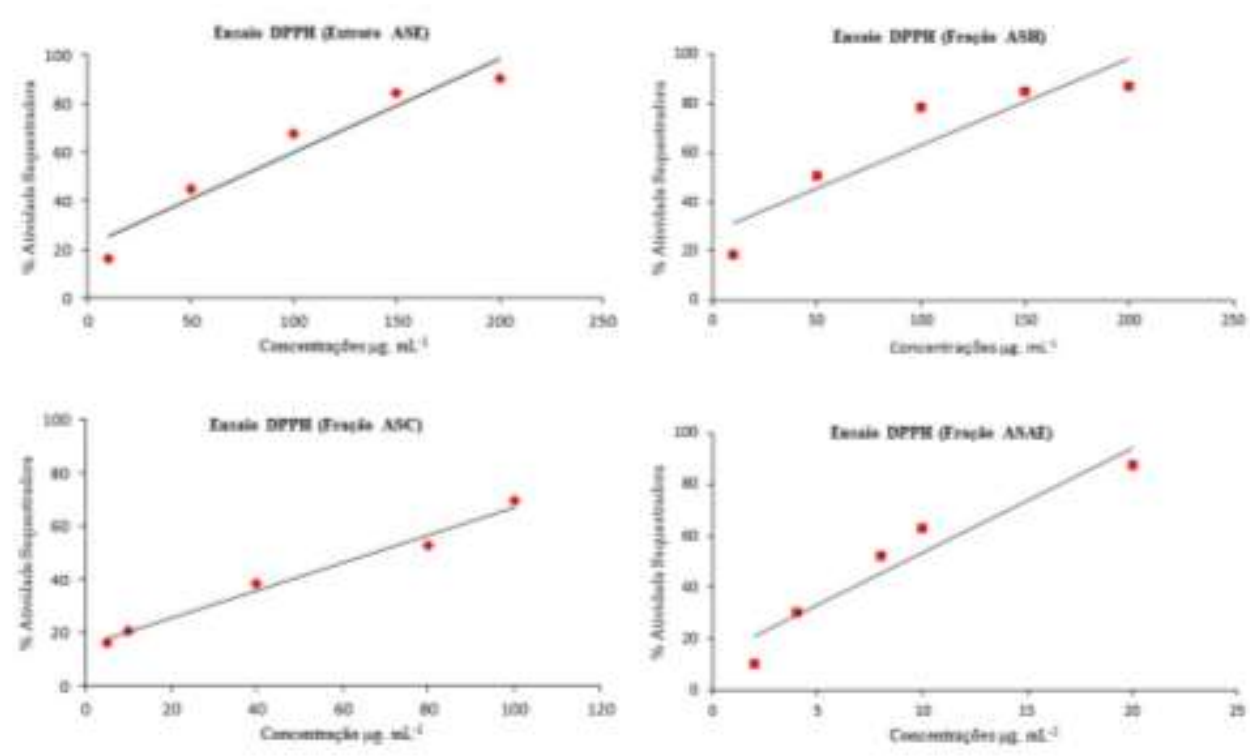

Fonte: Autores (2021).

\section{Conclusão}

Este é o primeiro estudo fitoquímico de folhas de A. sylvatica que resultou na identificação de flavonoides, alcaloides, saponinas, triterpenos e esteroides. Adicionalmente, relatamos o elevado teor de fenóis totais e a promissora atividade sequestradora de radicais livres no ensaio do DPPH de extrato e frações de folhas desta espécie. Estes dados justificam em parte as propriedades medicinais atribuídas a espécie, bem como, a importância desta frutífera como fonte de substâncias naturais capazes de captar radicais livres e atenuar seus efeitos deletérios no organismo. Os autores sugerem estudos fitoquímicos adicionais visando a identificação e/ou isolamento dos princípios ativos que possam estar relacionados a atividade antioxidante observada.

\section{Agradecimentos}

Os autores agradecem a Universidade Federal do Tocantins - UFT. 


\section{Referências}

Alves, C. Q., David, J. M., David, J. P., Bahia, M. V. \& Aguiar, R. M. (2010). Métodos para determinação de atividade antioxidante in vitro em substratos orgânicos. Química Nova, 33(10), 2202-2210.

Almeida, J. R. G. S., Araújo, C. S., Pessoa, C. O., Costa, M. P. \& Pacheco, A. G. M. (2014). Atividade antioxidante, citotóxica e antimicrobiana de Annona vepretorum Mart. (Annonaceae). Revista Brasileira de Fruticultura, 36, 258-264.

Araújo, R. C. P., Neves, F. A. R., Formagio, A. S. N., Kassuya, C. A. L., Stefanello, M. E. A., Souza, V. V., Pavan, F. R. \& Croda, J. (2014). Evaluation of the anti-mycobacterium tuberculosis activity and in vivo acute toxicity of Annona sylvatica. BMC Complementary and Alternative Medicine, 14(1), 209-219.

Azevedo, L. F. P.; Faria, T. S. A.; Pessanha, F. F.; Araújo, M. F. \& Lemos, G. C. S. (2014). Triagem fitoquímica e atividade antioxidante de Costus spicatus (Jacq.) S.W. Revista Brasileira de Plantas Medicinais, 16(2), 209-215.

Bessa, N. G. F. D., Borges, J. C. M., Beserra, F. P., Carvalho, R. H. A., Pereira, M. A. B., Fagundes, R., Campos, S. L., Ribeiro, L. U., Quirino, M. S.; Chagas Junior, A. F. \& Alves, A. (2013). Prospecção fitoquímica preliminar de plantas nativas do cerrado de uso popular medicinal pela comunidade rural do assentamento vale verde - Tocantins. Revista Brasileira de Plantas Medicinais, 15(4), 692-707.

Babu, M. M. \& Lawrence, B. (2014). Phytochemical analysis of Annona reticulata L. Leaf extracts. Applied Sciences, 4(5), 4-8.

Benites, R. S. R., Formagio, A. S. N., Argandoña, E. J. S., Volobuff, C. R. F., Trevizam, L. N. F., Vieira, M. C. \& Silva, M. S. (2015). Contents of constiuents and antioxidante activity of seed and pulp extracts of Annona coriacea and Annona sylvatica. Brazilian Journal of Biology, 75(3), 685-961.

Chen, S. L., Yu, H., Luo, H. M., Wu, Q., Li, C.-F. \& Steinmetz, A. (2016). Conservation and sustainable use of medicinal plants: problems, progress, and prospects. Chinese Medicine, 11, 37-47.

Carvalho Junior, A. R., Ferreira, R. O., Passos, M. S., Boeno, S. I. S., Virgens, L. L. G., Ventura, T.L.B.; Calixto, S.D., Lassounskaia, E., Carvalho, M. G., Braz-Filho, R. \& Vieira, I. J. C. (2019). Antimycobacterial and Nitric Oxide Production Inhibitory Activities of Triterpenes and Alkaloids from Psychotria nuda (Cham. \& Schltdl.) Wawra. Molecules, 24(6), 1026-1037.

Formagio, A. S. N., Vieira, M. C., Santos, L. A. C., Cardoso, C. A. L., Foglio, M. A., Carvalho, J. E., Silva, M. A. \& Kassuya, C. A. L. (2013). Composition and Evaluation of the Anti-Inflammatory and Anticancer Activities of the Essential Oil from Annona sylvatica A. St.-Hill. Journal of Medicinal Food, 16(1), $20-25$.

Ferreira, R. O., Carvalho Junior, A. R., Riger, C. J., Castro, R. N., Silva, T. M. S. \& Carvalho, M. G. (2016) Constituintes químicos e atividade antioxidante in vivo de flavonoides isolados de Clusia lanceolata (Clusiaceae). Química Nova, 39, 1093-1097.

Goncalves, G. L. P., Ribeiro, L. D., Gimenes, L., Vieira, P., Silva, M. F. D. F., Forim, M. R., Fernandes, J. B. \& Vendramim, J. D. (2015). Lethal and sublethal toxicities of Annona sylvatica (Magnoliales: Annonaceae) extracts to Zabrotes subfasciatus (Coleoptera: Chrysomelidae: Bruchinae). Florida Entomology, 98(3), 921-928.

Howes, M.-JR, Quave, C. L.; Collemare, J., et al. (2020). Molecules from nature: Reconciling biodiversity conservation and global healthcare imperatives for sustainable use of medicinal plants and fungi. Plants, People, Planet, 2, 463-481.

Ijaiya, I. S., Arzika, S., \& Abdulkadir, M. (2014). Extraction and Phytochemical Screening of the Root and Leave of Annona Senegalesis (Wild Custad Apple). Academic Journal of Interdisciplinary Studies, 3(7), 9-15.

Ibrahim, F.; Jaber, A.; Ibrahim, G.; Cheble, E. (2020). Antioxidant activity and total phenol content of different plant parts of Lebanese Annona Squamosa Linn. International Journal of Pharmacy and Pharmaceutical Sciences ,12(8), 100-105.

Lobão, A. Q., Silva, R. M. \& Forzza, R. C. (2012). Guatteria (Annonaceae) da Floresta Atlântica brasileira. Rodriguésia, 63(4), $1039-1064$.

Leite, D. O. D., Nonato, C. F. A., Camilo, C. J., Carvalho, N. K. G., Nobrega, M. G. L. A., Pereira, R. C. \& Costa J. G. M. (2020). Annona genus: traditional uses, phytochemistry and biological activities. Current Pharmaceutical Design, 26(33), 4056-4091.

Matos, F.T. (1997). Introdução à Fitoquímica Experimental. UFC.

Março, P. H. \& Poppi, R. J. (2008). Procedimentos analíticos para identificação de antocianinas presentes em extratos naturais. Química Nova, 31(5), 12181223 .

Mishra, S., Ahmad, S., Kumar, N., \& Sharma, B. K. (2013). Annona muricata (the cancer killer): a review. The Global Journal of Pharmaceutical Research, 2(1), 1613-1618.

Martins, G. V., Alves, D. R., Viera-Araújo, F. M., Rondon, F., Braz-Filho, R. \& Morais, S. M. (2018). Estudo Químico e Avaliação das Atividades Antioxidante, Antiacetilcolinesterase e Antileishmanial de Extratos de Jatropha gossypifolia L. (Pião Roxo). Revista Virtual de Química, 10(1), 21-36.

Neethu Simon K., Santhoshkumar R. \& Neethu S. K. (2016). Phytochemical analysis and antimicrobial activities of Annona squamosa (L) leaf extracts. Journal of Pharmacognosy and Phytochemistry. 5(4):128-131.

Newman, D. J. \& Cragg, G. M. (2020). Natural Products as Sources of New Drugs over the Nearly Four Decades from 01/1981 to 09/2019. Journal of Natural Products, 83(3),770-803.

Pinto, A. C. Q., Cordeiro, M. C. R., Andrade, S. R. M., Ferreira, F. R., Filgueiras, H. A. C., Alves, R. E. \& Kinpara, D. I. (2005). Annona species. International Centre for Underutilised Crops, University of Southampton. 
Research, Society and Development, v. 10, n. 15, e340101522811, 2021

(CC BY 4.0) | ISSN 2525-3409 | DOI: http://dx.doi.org/10.33448/rsd-v10i15.22811

Paz, W. H. P., Santos, R. P. \& Lima, F. L. (2014). Prospecção preliminar fitoquímica e avaliação da atividade antimicrobiana dos extratos de Cenostigma macrophyllum Tul. Boletim Informativo Geum, 5(4), 52-59.

Quílez, A. M., Fernández-Arche, M. A., García-Giménez, M. D., \& De la Puerta, R. (2018). Potential therapeutic applications of the genus Annona: Local and traditional uses and pharmacology. Journal of Ethnopharmacology, 225, 244-270.

Rabêlo, S. V., Costa, M. M., Libório, R. C. \& Almeida, J. R. G. S. (2014). Atividade antioxidante e antimicrobiana de extratos de atemoia (Annona cherimola Mill. X A. squamosa L.). Revista Brasileira de Fruticultura, 36, 265-271.

Simões, C. M. O., Schenkel, E. P., Mello, J. C. P., Mentz, L. A. \& Petrovick, P. R. (2016). Farmacognosia: do produto natural ao medicamento. Artmed.

Singleton, V. L., Orthorfer, R. \& Lamuela-Raventós, R. M. (1999). Analysis of total phenols and other oxidation substrates and antioxidants by means of folin-ciocalteu reagente. Methods in Enzymology, 299, 152-178.

Sousa, C. M. M., Silva, H. R., Vieira-Jr., G. M., Ayres, M. C. C., Costa, C. L. S., Araújo, D. S., Cavalcante, L. C. D., Barros, E. D. S., Araújo, P. B. M., Brandão, M. S. \& Chaves, M. H. (2007). Fenóis totais e atividade antioxidante de cinco plantas medicinais. Química Nova, 30, 351-355.

Vendruscolo, G. S. \& Mentz, L. A. (2006). Levantamento etnobotânico das plantas utilizadas como medicinais por moradores do bairro Ponta Grossa, Porto Alegre, Rio Grande do Sul. Iheringia, Série Botânica, 61(1-2), 83-103.

Vechia, L.D., Gnoatto, S.C.B. \& Gosmann, G. (2009) Derivados oleananos e ursanos e sua importância na descoberta de novos fármacos com atividade antitumoral, anti-inflamatória e antioxidante. Química Nova, 32, 1245-1252.

Vetrania, C., Costabile, G., Vitale, M. \& Giacco, R. (2020). (Poly)phenols and cardiovascular diseases: Looking in to move forward. Journal of Functional Foods, 71, 104013. 